\title{
Construction the evasion strategy in the differential game of many pursuers and one evader with integral constraints
}

\begin{abstract}
We consider an evasion differential game of many pursuers and one evader with integral constraints in the space R3. The game is described by simple equations. Control functions of the players are subjected to coordinate-wise integral constraints. Evasion is said to be possible if the state of the evader does not coincide with that of any pursuer. Strategy of the evader is constructed based on controls of the pursuers with lag. A sufficient condition of evasion from many pursuers is obtained and an illustrative example is provided.
\end{abstract}

Keyword: Control; Differential game; Evasion; Integral constraint; Strategy 